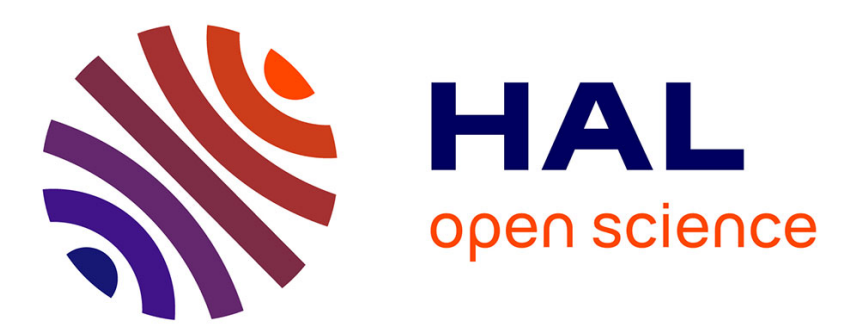

\title{
On the morphological stability of multicellular tumour spheroids growing in porous media \\ Chiara Giverso, Pasquale Ciarletta
}

\section{To cite this version:}

Chiara Giverso, Pasquale Ciarletta. On the morphological stability of multicellular tumour spheroids growing in porous media. European Physical Journal E: Soft matter and biological physics, 2016, 39 (10), pp.92. 10.1140/epje/i2016-16092-7 . hal-01383173

\section{HAL Id: hal-01383173 \\ https://hal.sorbonne-universite.fr/hal-01383173}

Submitted on 18 Oct 2016

HAL is a multi-disciplinary open access archive for the deposit and dissemination of scientific research documents, whether they are published or not. The documents may come from teaching and research institutions in France or abroad, or from public or private research centers.
L'archive ouverte pluridisciplinaire HAL, est destinée au dépôt et à la diffusion de documents scientifiques de niveau recherche, publiés ou non, émanant des établissements d'enseignement et de recherche français ou étrangers, des laboratoires publics ou privés. 


\title{
On the morphological stability of multicellular tumour spheroids growing in porous media
}

\author{
Chiara Giverso ${ }^{1}$ and Pasquale Ciarletta ${ }^{1,2 a}$ \\ 1 Dipartimento di Matematica - MOX, Politecnico di Milano, Piazza Leonardo da Vinci, 32 - 20133 Milano, Italy; \\ 2 CNRS and Sorbonne Universités, UPMC Univ Paris 06, UMR 7190, Institut Jean le Rond d'Alembert, 4 place Jussieu case \\ 162, 75005 Paris, France
}

Received: date / Revised version: date

\begin{abstract}
Multicellular tumour spheroids (MCTSs) are extensively used as in-vitro system models for investigating the avascular growth phase of solid tumours. In this work, we propose a continuous growth model of heterogeneous MCTSs within a porous material, taking into account a diffusing nutrient from the surrounding material directing both the proliferation rate and the mobility of tumour cells. At the time scale of interest, the MCTS behaves as an incompressible viscous fluid expanding inside a porous medium. The cell motion and proliferation rate are modelled using a non-convective chemotactic mass flux, driving the cell expansion in the direction of the external nutrients' source. At the early stages, the growth dynamics is derived by solving the quasi-stationary problem, obtaining an initial exponential growth followed by an almost linear regime, in accordance with experimental observations. We also perform a linear-stability analysis of the quasi-static solution in order to investigate the morphological stability of the radially symmetric growth pattern. We show that mechano-biological cues, as well as geometric effects related to the size of the MCTS subdomains with respect to the diffusion length of the nutrient, can drive a morphological transition to fingered structures, thus triggering the formation of complex shapes that might promote tumour invasiveness. The results also point out the formation of a retrograde flow in the MCTS close to the regions where protrusions form, that could describe the initial dynamics of metastasis detachment from the in-vivo tumour mass. In conclusion, the results of the proposed model demonstrate that the integration of mathematical tools in biological research could be crucial for better understanding the tumour's ability to invade its host environment.
\end{abstract}

PACS. 87.18.Hf Spatiotemporal pattern formation in cellular populations - 87.18.Gh Cell-cell communication; collective behavior of motile cells $-46.32 .+\mathrm{x}$ Static buckling and instability

\section{Introduction}

A multicellular tumour spheroid (MCTS) is an ensemble of tumour cells organized in a multi-layered structure ${ }^{1,2}$. In general, a MCTS consists of a central core of necrotic cells, surrounded by a layer of quiescent (i.e. dormant) cells and an outer rim of proliferating cells ${ }^{1-4}$.

MCTSs are widely used in vitro to study the early stages of avascular tumour growth and to assess the efficacy of anti-cancer drugs and therapies, since their growth and structure resemble the in vivo avascular phase of solid tumour invasion. Such an early growth phase is characterized by diffusion-limited growth, since the tumour absorbs vital nutrients via diffusion from the external environment ${ }^{1,3,5}$. Thus, diffusion may become suddenly ineffective in the center of the tumour mass, forming a characteristic necrotic core (see Fig. 1-a). At later stages, a solid tumour is characterized by the occurrence of angiogenesis

\footnotetext{
a e-mail: pasquale.ciarletta@polimi.it
}

(i.e. the process by which the tumour induces new blood vessels formation from the nearby existing vasculature), thus switching to a vascular growth phase ${ }^{6,7}$.

The analysis of the avascular growth phase in tumours has attracted a lot of interest in the mathematical and physical research communities, and a large number of in silico mathematical models has been proposed ${ }^{2,8-16}$. Thanks to the controllability and the reproducibility of the experimental setting, MCTS has become a widely used system model for the development of theoretical models.

The classical approach of deterministic tumour model comprised an ordinary differential equation (ODE), derived from either mass conservation or population dynamics, coupled with at least one reaction-diffusion equation, representing the spatio-temporal distribution of vital nutrients or chemical signals inside the tumour ${ }^{2,9-12,14,15,17}$. Only recently, many authors have extended such models including the pivotal role of mechanics in tumour growth. In most cases, fluid-like constitutive equations have been used to model the tumour mass ${ }^{18-26}$. This choice is obvi- 
ously only an approximation of the by far more complex behaviour of cellular aggregates, that also display solidlike properties related to the adhesive characteristics of cells ${ }^{27}$ and to the mechanical properties of the single cell in the cluster. Thus, in some limiting cases, cell aggregates are better described as solids with linear or eventually nonlinear elasticity, in which compressive and shear loads are balanced by the solid stress in the body, depending on the strain of its material points ${ }^{28-33}$. A solid-like constitutive equation has been advocated for its suitability of accounting for both residual stresses ${ }^{29,32,34}$ and the plastic behaviour of cellular aggregates ${ }^{35-37}$. Even though these considerations support the idea that a cellular aggregate can behave as a solid at some extent, experimental evidences ${ }^{26,38,39}$ have shown that aggregates behave as elastic solids on short timescales (of the order of a few minutes) but display a fluid-like behaviour at longer times. Furthermore, it was shown that cellular aggregates behave as an elastic solids at time scales short compared to that of cell division and apoptosis, and as a fluid (with the traceless stress that relaxes to zero) for long times ${ }^{40}$. Thus the description of MCTSs as a liquid is widespread.

Even though the existing mathematical models on both solid tumours and MCTSs successfully reproduce the experimentally observed growth dynamics ${ }^{2,9-12,14,15,17,41,42}$ they poorly consider the mechanical and chemical interaction with the surrounding environment. Furthermore, most approaches assume that the initial spherical symmetry is preserved during the growth of the aggregate ${ }^{28-30}$, whilst only in few cases ${ }^{11,12,15}$ the development of tumour irregular contours has been taken into account. Indeed, it is known that some solid tumours, e.g. carcinomas, grow almost spherically only in the first stages of their progression ${ }^{1,3,5}$, while they might show a less defined and even asymmetric outer boundary $^{43}$ (see Fig. 1-b). Since higher irregular contours usually indicate aggressive tumours, the capability to undergo a morphological transition might promote tumour infiltration and invasion within the surrounding tissue $2,11,12,15,44-46$. Thus, it has been proposed that some measure of the irregularity of a tumour boundary (e.g. its fractal index measured via particular medical imaging techniques such as computerized tomography scans), may provide clinicians with useful information for its prognosis and treatment ${ }^{44-46}$, being potentially useful in predicting the efficacy of drug treatment or chemother$\operatorname{apy}^{47,48}$.

In this work we go beyond the state-of-the art in the field ${ }^{2,49,50}$ by proposing a mathematical model that accounts for the presence of a surrounding porous media with a finite thickness. Thus, nutrient diffusion from the external environment creates a chemical gradient that directs both the proliferation rate and the motility of the tumour cells. MCTS is modelled as a viscous fluid with adhesive interactions at the border, expanding inside a porous material.

This work is organized as follows. First, we introduce in Section 2 the mathematical model describing the expansion of an initially spherical tumour. In Section 3, we derive the radially-symmetric solution of the quasi-stationary

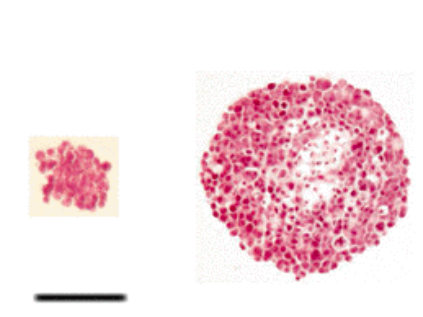

$100 \mu \mathrm{m}$

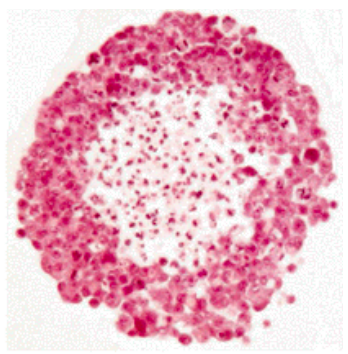

(a)
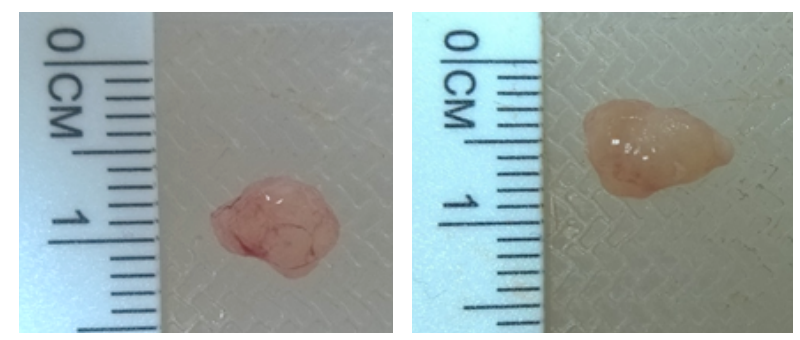

(b)

Figure 1. (a) Morphological evolution of a multicellular tumour spheroid of HeLa cells, showing the development of an undulated contour and a necrotic core (reproduced with permission from ${ }^{51}$ ). HeLa cells were trypsinized, counted and grown as multicellular spheroids using the liquid overlay technique. The sections were counterstained with hematoxylin and eosin to visualize the cytoplasms of the cells. The multicellular spheroid section is reproduced at days 0,4 and 12 , from left to right. (b) Solid tumours extracted from mice after orthotopic implant of MCF10CA1a cell lines in the mammary fat pad of the nude mice (courtesy of T. Stylianopoulos, Cancer Biophysics Laboratory, University of Cyprus).

problem. Then, we perform a linear stability on the quasistatic tumour growth. Finally, in Section 4, we discuss the modelling results with respect to the key chemo-mechanical and geometric parameters that govern the mathematical problems, highlighting the key mechano-biology effects that promote a morphological transition during tumour invasion.

\section{Mathematical Model}

The MCTS is modelled as a three dimensional continuum growing inside a rigid porous structure, representing the surrounding environment, usually extracellular matrix (ECM) or matrigel. In this respect, the proposed model refers to the in vitro case in which MCTS grows inside a three dimensional either natural medium (e.g. agarose gel, hyaluronic acid gel) or synthetic matrices scaffolds (e.g. polylactide and polyglycolide biodegradable structures mimicking a tissue-like environment) ${ }^{52}$.

The outer boundary of the tumour is considered as a freely moving material interface separating the tumour cells from the surrounding medium. 
In particular, we account for the presence of a central region of necrotic cells, surrounded by a layer of quiescent and proliferating cells. Thus, the whole domain $\Omega$ is divided in different regions, depending on the residing cellular population (see Fig. 2):

- the necrotic cells are located in the central core of the spheroid, in a region called $\Omega_{N}(t)$, with

$$
\Omega_{N}(t)=\left\{(r, \theta): r<R_{N}(t), 0<\varphi \leq \pi, 0<\theta \leq 2 \pi\right\},
$$

where $R_{N}$ is the radius of the necrotic core, that might evolve in time;

- the proliferative and quiescent tumour cells are located in the region

$$
\Omega_{T}(t)=\left\{(r, \theta): R_{N}(t)<r<R_{T}(t), 0<\varphi \leq \pi, 0<\theta \leq 2 \pi\right\},
$$

where $R_{T}$ is the radius of the spheroid, whose evolution in time represents the growth of the MCTS;

- the healthy space, composed by either the in vitro scaffold or the extracellular matrix, the extracellular liquid and possibly healthy cells (in vivo),

$$
\Omega_{H}(t)=\left\{(r, \theta): R_{T}(t)<r<R_{\text {out }}, 0<\varphi \leq \pi, 0<\theta \leq 2 \pi\right\},
$$

being $R_{\text {out }}$ the outer boundary of the whole domain.

The boundary between the necrotic core and the proliferative region is called $\partial \Omega_{N}(t)$, whereas the moving interface between the tumour region and the healthy space is denoted with $\partial \Omega_{T}(t)$. In the following we will consider that the interior boundary between the necrotic core and the quiescent-proliferative region does not evolve in time, since we are interested only in the evolution of the MCTS boundary, which is related to tumour infiltration inside the healthy region. Furthermore, we assume that the porous material is homogeneously distributed in the whole region $\Omega=\Omega_{N} \cup \Omega_{T}(t) \cup \Omega_{H}(t)$ and it is neither produced/degraded (i.e. behaves as inert matter), nor deformed (i.e. structurally rigid) by the moving tumour cells. We will consider a single nutrient species (e.g. oxygen) with volume concentration $n(\mathbf{x}, t)$, diffusing from the fixed outer boundary $\partial \Omega$ through the porous material. Thus, we assume that the vascular network providing the source of nutrients is outside the modelled domain, and can be represented by a boundary term at $\partial \Omega$. The diffusion coefficient is a constant value $D_{n}$ everywhere, but the nutrient is only consumed, with an uptake rate $\gamma_{n}$, in the region occupied by the proliferative and quiescent cells. Indeed we consider that the consumption of nutrients in the healthy region is negligible. This is certainly the case of MCTS growing inside artificial/natural scaffolds, but, in a first approximation, it can be used also to model the in vivo condition $^{20,53}$, since the net consumption of nutrients in the extracellular healthy space is negligible compared to the uptake by tumor cells 54 .

Thus, the 3D homogenized concentration per unit volume of this generic chemical species, indicated with $n(\mathbf{x}, t)$, obeys the following reaction-diffusion equation

$$
\dot{n}(\mathbf{x}, t)= \begin{cases}D_{n} \nabla^{2} n(\mathbf{x}, t) & \text { in } \Omega_{N}, \\ D_{n} \nabla^{2} n(\mathbf{x}, t)-\gamma_{n} n(\mathbf{x}, t) & \text { in } \Omega_{T}(t), \\ D_{n} \nabla^{2} n(\mathbf{x}, t) & \text { in } \Omega_{H}(t) .\end{cases}
$$

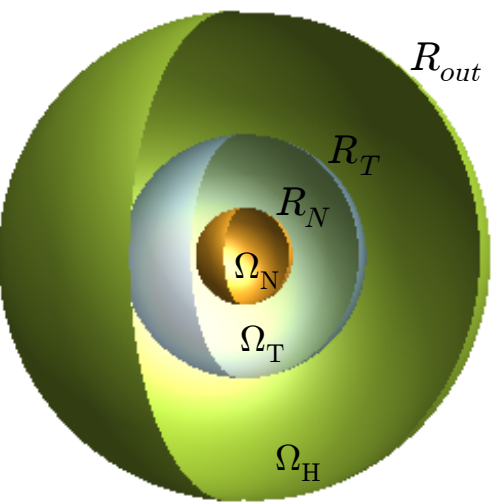

Figure 2. Representation of the domain used for the analytical analysis. At time $t=0$, the three domains $\Omega_{N}, \Omega_{T}$ and $\Omega_{H}$ are concentric spherical shells, with radius $R_{N}, R_{T}$ and $R_{\text {out }}$, respectively. In this work, we consider that only the tumour boundary $\partial \Omega_{T}$ evolves in time.

We remark that, in principle, the uptake rate $\gamma_{n}$ should depend on the tumour cell density, although, in the following, it will be considered homogeneous and constant over time. Even the diffusion coefficient $D_{n}$ can be affected by the cell packing inside the tumor and by the extracellular matrix alignment and distribution. However, coherently with the hypothesis of an inert, rigid and homogeneous extracellular matrix distributed in the whole domain, the diffusion of nutrients can be assumed to be constant ${ }^{53,55}$. The diffusing nutrient notably not only affects the growth of single individuals in the tumour but also directs cell movements, e.g. through chemotaxis ${ }^{56,57}$. Therefore, we consider a non-convective mass flux term, m, taking into account both tumour proliferation and chemotactic motion, differently from the standard volumetric production rate considered in literature ${ }^{2,11-15}$. Accordingly, the mass balance inside $\Omega_{T}(t)$ reads

$$
\frac{d \rho}{d t}+\rho \nabla \cdot \mathbf{v}=\nabla \cdot \mathbf{m} \quad \text { in } \Omega_{T}(t)
$$

where $\rho$ is the tumour cell density, which is approximately the same of water. Since mass transport phenomena in MCTSs are driven by the local concentration of chemicals, the mass flux vector appearing in Eq. (2) should depend on nutrient availability. A simple constitutive law for $\mathbf{m}$ can be taken in the form of a chemotactic term ${ }^{56,58}$, i.e. $\mathbf{m}=\chi \rho \nabla n$, where $\chi$ is the chemotactic coefficient, here considered constant. Consequently, the mass flux $\mathbf{m}$ describes the expansion of the tumour due to proliferation and driven by chemotaxis towards higher concentration of nutrients.

Assuming that the living aggregate can be macroscopically modelled as a Newtonian fluid, Darcy's law describes its motion inside the inert, porous surrounding medium ${ }^{2,18}$. Thus, the cell velocity $\mathbf{v}$ is related to the pressure field $p$ 
through

$$
\mathbf{v}=-K_{p} \nabla p,
$$

where $K_{p}$ is related to the permeability of the medium, $k$, and the viscosity of the cellular material, $\mu$, by $K_{p}=k / \mu$. Assuming the incompressibility of the cellular spheroid, which is mostly composed by water, we impose $d \rho / d t=0$ in Eq. (2), so that the relation between the pressure $p$ and the nutrient concentration $n$ reads

$$
\nabla^{2} p=-\frac{\chi}{K_{p}} \nabla^{2} n \text { in } \Omega_{T}(t),
$$

which has been obtained substituting the Darcy's law (3) and the constitutive relations for $\mathbf{m}$ in the mass balance equation (2). In summary, the coupling of Eq. (1) with Eq.(4), complemented by a proper set of boundary conditions (BCs), describes the macroscopic evolution of the avascular tumour inside the healthy tissue.

In particular, for the pressure we impose the Young-Laplace equation at the moving boundary $\partial \Omega_{T}(t)$ and the null velocity of the tumour cells at the fixed boundary $\partial \Omega_{N}$, i.e.

$$
\begin{aligned}
p & =p_{0}-\sigma_{b} C \quad \text { on } \partial \Omega_{T}(t), \\
\mathbf{v}_{\partial \Omega_{N}} \cdot \mathbf{n}_{N}=0 & \left.\rightarrow(\nabla p)\right|_{\partial \Omega_{N}} \cdot \mathbf{n}_{N}=0 \quad \text { on } \partial \Omega_{N},
\end{aligned}
$$

being $\mathbf{n}_{N}$ the normal at the fixed boundary $\partial \Omega_{N}, C$ the local curvature of the free boundary $\partial \Omega_{T}(t), p_{0}$ the constant pressure in the outer healthy domain and $\sigma_{b}$ the surface tension at the moving interface. The surface tension $\sigma_{b}$ arises from the collective adhesive interaction among tumour cells at the MCTS boundary, primarily mediated by cadherins, ${ }^{59}$ and from the differential contractility between the cell-cell and cell-medium interfaces, mainly mediated by $\alpha$-catenin ${ }^{60}$. Even if, in principle the surface tension $\sigma_{b}$ depends on the density of cells, the distribution of cadherins and the presence of $\alpha$-catenin ${ }^{60}$, we will assume that it can be considered constant, for the chosen cellular population composing the aggregate.

For what concerns the chemical species, in absence of an interfacial structure, the continuity for the nutrient concentration and flux can be assumed (both in $\partial \Omega_{T}(t)$ and in $\partial \Omega_{N}$ ), and the concentration at the outer boundary can be assumed constant (to model the source of nutrients from the external vascular network), so that

$$
\begin{aligned}
\left.n\right|_{\partial \Omega} & =n_{\text {out }} & & \text { on } \partial \Omega, \\
\left.\llbracket n \rrbracket\right|_{\partial \Omega_{T}}=0,\left.\quad \llbracket \nabla n \rrbracket\right|_{\partial \Omega_{T} \cdot \mathbf{n}} & =0 & & \text { on } \partial \Omega_{T}, \\
\left.\llbracket n \rrbracket\right|_{\partial \Omega_{N}}=0,\left.\llbracket \nabla n \rrbracket\right|_{\partial \Omega_{N}} \cdot \mathbf{n}_{N} & =0 & & \text { on } \partial \Omega_{N},
\end{aligned}
$$

where $\mathbf{n}$ is the outward normal vector at the boundary $\partial \Omega_{T}$ and $\left.\llbracket(\cdot) \rrbracket\right|_{\partial \Omega_{j}}$ denotes the jump of the quantity between brackets across the boundary $\partial \Omega_{j}$, with $j=N, T$.

Finally, the compatibility condition at the free interface imposes

$$
\frac{\mathrm{d} \mathbf{x}_{\partial \Omega_{T}}}{\mathrm{~d} t} \cdot \mathbf{n}=\mathbf{v}_{\partial \Omega_{T}} \cdot \mathbf{n} \quad \text { on } \partial \Omega_{T} .
$$

In the following we will work with dimensionless equations, obtained writing the system of Eqs. (1)-(4) in terms of the dimensionless chemical concentration, $\bar{n}=n / n_{c}$, and the dimensionless pressure, $\bar{p}=p / p_{c}$ and referring to the geometry outlined in Fig. 2. The dimensionless quantities are obtained using the following characteristic time $t_{c}$, length $l_{c}$, velocity $v_{c}$, pressure $p_{c}$ and chemical concentration $n_{c}: t_{c}=\gamma_{n}^{-1}, l_{c}=\sqrt{D_{n} \gamma_{n}^{-1}}, v_{c}=\sqrt{D_{n} \gamma_{n}}, p_{c}=D_{n} K_{p}^{-1}$, $n_{c}=n_{\text {out }}$. Finally, the resulting dimensionless systems of equations reads

$$
\begin{aligned}
& \dot{\bar{n}}=\left\{\begin{array}{lc}
\bar{\nabla}^{2} \bar{n} & \text { for } \bar{r}<\bar{R}_{N} \\
\bar{\nabla}^{2} \bar{n}-\bar{n} & \text { for } \bar{R}_{N}<\bar{r}<\bar{R}_{T}(t) \\
\bar{\nabla}^{2} \bar{n} & \text { for } \bar{R}_{T}(t)<\bar{r}<\bar{R}_{\text {out }}
\end{array}\right. \\
& \bar{\nabla}^{2} \bar{p}=-\beta \bar{\nabla}^{2} \bar{n} \quad \text { for } \bar{R}_{N}<\bar{r}<\bar{R}_{T}(t) \\
& \left.\llbracket \bar{n} \rrbracket\right|_{\bar{R}_{N}}=0,\left.\llbracket \bar{\nabla} \bar{n} \rrbracket\right|_{\bar{R}_{N}} \cdot \overline{\mathbf{n}}_{N}=0,(\bar{\nabla} \bar{p}) \cdot \overline{\mathbf{n}}_{N}=0 \\
& \text { for } \bar{r}=\bar{R}_{N} \\
& \left.\llbracket \bar{n} \rrbracket\right|_{\bar{R}_{T}}=0,\left.\llbracket \bar{\nabla} \bar{n} \rrbracket\right|_{\bar{R}_{T}} \cdot \overline{\mathbf{n}}=0, \bar{p}=\bar{p}_{0}-\bar{\sigma} \bar{C} \\
& \text { for } \bar{r}=\bar{R}_{T}(t) \\
& \bar{n}\left(\bar{t}, \bar{R}_{\text {out }}\right)=1 \quad \text { for } \bar{r}=\bar{R}_{\text {out }} \\
& \frac{\mathrm{d} \overline{\mathbf{x}}_{\bar{R}_{T}}}{\mathrm{~d} \bar{t}} \cdot \overline{\mathbf{n}}=\overline{\mathbf{v}}_{\bar{R}_{T}} \cdot \overline{\mathbf{n}}=-\left.\bar{\nabla} \bar{p}\right|_{\bar{R}_{T}} \cdot \overline{\mathbf{n}} \quad \text { for } \bar{r}=\bar{R}_{T}(t)
\end{aligned}
$$

The nondimensionalization leads to the definition of five dimensionless parameters, classified into two broad categories:

- $\beta:=\chi n_{c} / D_{n}$ and $\sigma:=\sigma_{b} K_{p} \gamma_{n}^{1 / 2} D_{n}^{-3 / 2}=\sigma_{b} K_{p} l_{c}^{-1 / 2} D_{n}^{-1}{ }^{234}$ define mechano-biology effect on the aggregate expan- ${ }_{235}$ sion, and are called motility parameters;

- $\bar{R}_{N}, \bar{R}_{T}$ and $\bar{R}_{\text {out }}$ (i.e. the dimensionless radii of the necrotic core, of the MCTS and the whole domain, respectively) define the geometrical properties of the system with respect to the diffusive length $l_{c}$, and are denoted as size parameters.

In particular, the dimensionless parameter $\beta$ represents the chemical effects associated to the expansion of MCTSs, since it can be regarded as the ratio between the typical time-scales of mass production over nutrient diffusion. On the other hand, the parameter $\sigma$ defines the influence of mechanical cues over tumour development, representing the ratio of the surface tension of the aggregate over the characteristic viscous pressure of the fluid ensemble. For sake of simplicity, in the following we will omit the barred notation to denote dimensionless quantities, e.g. $R_{T}$ stands for $\bar{R}_{T}$ and so on.

\section{Linear stability analysis of the quasi-static solution}

In this Section, we first derive the quasi-static solution of the proposed model in order to mimic the early avascular 
growth. We later perform a linear stability analysis to investigate the occurrence of a morphological instability at later growth stages.

\subsection{Quasi-stationary solution}

At early stages of avascular growth MCTSs maintain a spherical shape ${ }^{1,3,5}$. Thus, we look for a radially symmetric quasi-stationary solution, assuming that the diffusive process is much faster than the MCTS expansion, so that it is possible to drop the time derivative in Eq. (11a). This assumption is valid in many biological conditions, since a fast-growing tumour may expand at a rate of up to $0.5 \mathrm{~mm}$ /day, whereas a typical diffusion time scale is about 1 min (considering a typical length scale $L \approx 10^{-2} \mathrm{~cm}$ and a typical diffusion coefficient $\left.D \approx 10^{-6} \mathrm{~cm}^{2} \mathrm{~s}^{-1}\right)^{11}$. Thus, it is clear that the diffusion timescale of nutrients is much shorter than the growth timescale, so that the quasi-stationary assumption can be effectively formulated Furthermore for such long time scale the MSC can be actually treated as a viscous fluid.

Specializing our analysis to the case of a spherical tumour of radius $R_{T}$, we will denote with $n^{*}=n^{*}(r, t)$ the quasi-stationary solution of Eq. 11a and with $p^{*}=$ $p^{*}(r, t)$ the quasi-stationary pressure field satisfying (11b). Given the boundary conditions (11c)-(11d)-(11e) and considering that $n^{*}$ and $p^{*}$ should be bounded, the quasistationary fields read

$n^{*}=\left\{\begin{array}{lr}\frac{2 R_{\text {out }} e^{R_{T}+R_{N}}}{e^{2 R_{T}} w_{T}^{+}-e^{2 R_{N}} w_{T}^{-}} & \text {if } r \leq R_{N} \\ -\frac{e^{2 R_{N}}\left[e^{2\left(r-R_{N}\right)}\left(R_{N}+1\right) R_{\text {out }}+\left(R_{N}-1\right) R_{\text {out }}\right]}{r w_{T}^{-} e^{r-R_{T}+2 R_{N}}-r w_{T}^{+} e^{r+R_{T}}} & \text { if } R_{N}<r \leq R_{T}(1 \\ \frac{R_{\text {out }}\left[e^{2 R_{T}}\left(R_{N}+1\right)\left(r-R_{T}+1\right)-e^{2 R_{N}}\left(R_{N}-1\right)\left(r-R_{T}-1\right)\right]}{r\left(e^{2 R_{T}} w_{T}^{+}-e^{2 R_{N}} w_{T}^{-}\right)} & \text {otherwise }\end{array}\right.$

$p^{*}=p_{0}+\frac{\sigma}{R_{T}}+\beta\left(n_{R_{T}}^{*}-n^{*}\right)$,

where we called $n_{R_{T}}^{*}=n^{*}\left(R_{T}\right)$ the concentration of the nutrient at the boundary of the aggregate and we defined $w_{T}^{+}=\left(R_{N}+1\right)\left(R_{\text {out }}-R_{T}+1\right)$ and $w_{T}^{-}=\left(R_{N}-1\right)\left(R_{\text {out }}-\right.$ $\left.R_{T}-1\right)$, being $w_{T}=\left(R_{\text {out }}-R_{T}\right)$ the width of the region occupied by the tumour. Then, using Eq. (11f), it is possible to compute the quasi-stationary velocity of the front, which is directed along the radial direction for symmetry considerations, i.e. $\mathbf{v}^{*}=v_{r}^{*} \mathbf{e}_{r}$, with

$$
v_{r}^{*}\left(R_{T}\right)=\beta \frac{R_{\text {out }}\left(e^{2 R_{N}}\left(R_{N}-1\right)\left(R_{T}+1\right)-\left(R_{N}+1\right) e^{2 R_{T}}\left(R_{T}-1\right)\right)}{R_{T}^{2}\left(e^{2 R_{N}}\left(R_{N}-1\right)\left(w_{T}^{-}\right)-\left(R_{N}+1\right) e^{2 R_{T}}\left(w_{T}^{+}\right)\right)} .
$$

Equation (14) can be integrated numerically to determine the evolution of the spheroid border over time. The result, reported in Fig. 3 for different value of the parameter $\beta$, highlights the existence of an initial phase in which the growth of the aggregate is nearly exponential and a subsequent one in which the expansion of the tumour is almost linear, as observed in ${ }^{32,61}$. Indeed, in standard MCTS free-growth (i.e. without the introduction of ${ }_{3236}^{325}$ external stress) in liquid suspension or at moderate agarosis gel concentration, the plot of the tumor diameter over

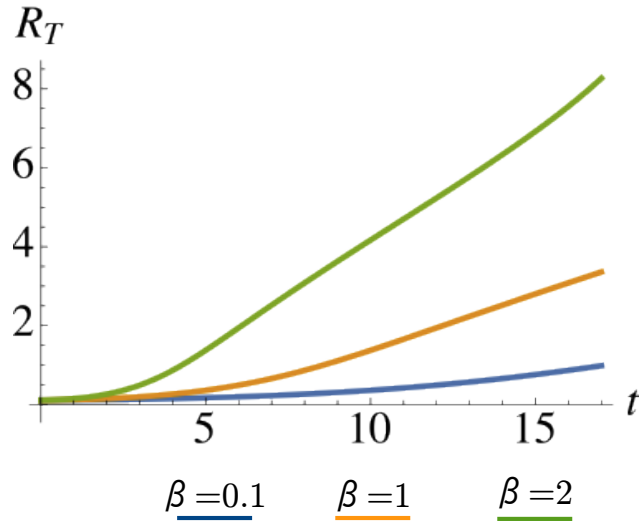

Figure 3. Quasi-stationary solution of the proposed model, depicting the radius of the tumour over time for different values of the motility parameter $\beta$. At early stages the growth is exponential, as a consequence of the bulk availability of nutrients. At later stages, the growth law is almost linear, reflecting the higher nutrient concentration on the outer surface of the growing spheroid.

time exhibits an early stage of exponential growth, corresponding to spheroid volumetric growth, since nutrients are available everywhere in the spheroid bulk ${ }^{32,61}$. Subsequently, when the diameter of the spheroid becomes much larger than the penetration length of the nutrient, the cellular growth becomes mainly localized on the surface of the tumor, leading to a linear growth in time.

\subsection{Perturbation of the quasi-stationary solution}

In this paragraph, we investigate the stability of the steady, radially-symmetric solution by applying small perturbations of the MCTS boundary.

Let $R_{T}^{*}$ be the unperturbed position of the moving interface, we consider a small perturbation $(\varepsilon \ll 1)$ of the kind

$$
R(\theta, \varphi, t)=R_{T}^{*}(t)+\varepsilon e^{\lambda t} \mathbb{R e}\left[\mathrm{Y}_{\ell}^{\mathrm{m}}(\theta, \varphi)\right] .
$$

where $\lambda \in \mathbb{R}$ is the amplification rate (or time-growth rate) of the perturbation and $Y_{\ell}^{m}(\theta, \varphi)$ is the spherical harmonic of degree $\ell$ and order $m$, with $m \in \mathbb{N}, \ell \in \mathbb{N}^{+}$and $|m| \leq \ell$. The spherical harmonics $Y_{\ell}^{m}(\theta, \varphi)$ form a complete set of orthonormal functions and thus any square-integrable function can be expanded as a linear combination of spherical harmonics. For physical consistency, the variations of $n$ and $p$ from the quasi-stationary solutions $n^{*}$ and $p^{*}$ should be in the form

$$
\begin{aligned}
& n(r, \theta, \varphi, t)=n^{*}(r, t)+\varepsilon n_{1}(r) e^{\lambda t} \mathbb{R e}\left[\mathrm{Y}_{\ell}^{\mathrm{m}}(\theta, \varphi)\right] \\
& p(r, \theta, \varphi, t)=p^{*}(r, t)+\varepsilon p_{1}(r) e^{\lambda t} \mathbb{R e}\left[\mathrm{Y}_{\ell}^{\mathrm{m}}(\theta, \varphi)\right],
\end{aligned}
$$

Using Eq. (11a) and the relation $\nabla_{\Omega}^{2} Y_{\ell}^{m}+\ell(\ell+1) Y_{\ell}^{m}=0$, where we set the angular part of the Laplacian operator as $\nabla_{\Omega}^{2}(\cdot)=1 / \sin \theta \partial / \partial \theta(\sin \theta \partial(\cdot) / \partial \theta)+1 / \sin ^{2} \theta \partial^{2}(\cdot) / \partial \phi^{2}$, the term $n_{1}$ must obey the following ODE

$$
r^{2} n_{1}^{\prime \prime}(r)+2 r n_{1}^{\prime}(r)-\left(\ell(\ell+1)+\left(\lambda+\mathbf{1}_{\Omega_{T}}\right) r^{2}\right) n_{1}(r)=0,
$$


where primes denote derivatives on $r$ and $\mathbf{1}_{\Omega_{T}}=1$ if $R_{N}<r \leq R_{T}^{*}, \mathbf{1}_{\Omega_{T}}=0$ otherwise. The solution of Eq. (18), for $\lambda \neq\{0,-1\}$ is

$$
n_{1}(r)= \begin{cases}C_{1} i_{\ell}(\sqrt{\lambda} r) & \text { if } r \leq R_{N} \\ B_{1} i_{\ell}(\sqrt{\lambda+1} r)+B_{2} k_{\ell}(\sqrt{\lambda+1} r) & \text { if } R_{N}<r \leq R_{T}^{*}(19) \\ A_{1} i_{\ell}(\sqrt{\lambda+1} r)+A_{2} k_{\ell}(\sqrt{\lambda+1} r) & \text { if } R_{T}^{*}<r \leq R_{\text {out }},\end{cases}
$$

where $i_{\ell}(r)$ and $k_{\ell}(r)$ are the modified spherical Bessel function of the first and second kind, respectively, evaluated in $r$. The coefficients $A_{1}, A_{2}, B_{1}, B_{2}, C_{1}$ appearing in the expression of $n_{1}(r)$ can be determined imposing the incremental boundary conditions for the concentration field (11c), (11d) and (11e), being

$$
\begin{array}{r}
\left.\llbracket n_{1} \rrbracket\right|_{R_{N}}=0,\left.\quad \llbracket \frac{\partial n_{1}}{\partial r} \rrbracket\right|_{R_{N}}=0, \\
\left.\llbracket n_{1} \rrbracket\right|_{R_{T}^{*}}=0,\left.\quad \llbracket \frac{\partial n_{1}}{\partial r} \rrbracket\right|_{R_{T}^{*}}=n_{0}, n_{1}\left(R_{\text {out }}\right)=0 .
\end{array}
$$

The perturbed pressure field $p_{1}$ in $\Omega_{T}$ is obtained from Eq.(11b) that leads to

$$
p_{1}(r)=Q r^{\ell}+W r^{-\ell-1}-\beta\left(B_{1} i_{\ell}(\sqrt{\lambda+1} r)+B_{2} k_{\ell}(\sqrt{\lambda+1} r)\right)
$$

where the constants $Q$ and $W$ can be determined from the boundary conditions on the pressure field (11c) and (11d), considering only the first order terms, i.e.

$$
p_{1}\left(R_{T}^{*}\right)=-\sigma \frac{2}{R_{T}^{* 2}}(2-(\ell+1) \ell)-\left.\frac{\partial p^{*}}{\partial r}\right|_{R_{T}^{*}},\left.\frac{\partial p_{1}}{\partial r}\right|_{R_{N}}=0
$$

perturbation theory ${ }^{62}$ Finally, using standard procedures in perturbation theory ${ }^{62}$, imposing the boundary condition (10) at the perturbed interface and neglecting the terms of order higher than the first in the series expansion, it is possible to obtain the following dispersion equation

$$
\lambda=-p^{* \prime \prime}\left(R_{T}^{*}\right)-p_{1}^{\prime}\left(R_{T}^{*}\right),
$$

which has the same form of the relation found for the rectilinear front on an infinite domain ${ }^{63}$ or an expanding circular colony ${ }^{64,65}$. The dispersion equation (24) is an implicit function of the time-growth mode $\lambda$ and the spherical harmonic degree $\ell$, depending on the five dimensionless parameters $\beta_{i}, \sigma, R_{N}, R_{T}^{*}$ and $R_{\text {out }}$. Interestingly, $\lambda$ does not depend on the azimuthal component of the model solutions $Y_{\ell}^{m}(\phi, \theta)$, i.e. the solutions are independent of the order $m$, as observed also in previous works based on different models ${ }^{15,50}$.

\section{Results and Discussion}

The dispersion equation (24) has been solved numerically in order to investigate the global stability of the solutions depending on the system parameters. The corresponding dispersion diagrams are reported in Fig. 4 for differents values of both the size and the motility parameters. Ass in classical perturbation theory ${ }^{62}$, a positive real part of the growth rate $\lambda$ implies global instability, thus highlight a critical spatial mode of the perturbation defined by the degree $\ell$ associated with the highest positive growth rate. Interestingly, Fig. 4 shows that the spheroid front is linearly unstable at small $\ell$, with $\ell=1$ being always unstable. Indeed, whilst for a spheroid growing inside an infinite homogeneous domain with constant chemical concentration, one would expect to find $\lambda=0$ for $\ell=1$, due to translational symmetry ${ }^{11,15,50}$, we must remind that in our case, due to the presence of the external environment the translational symmetry is no longer preserved.

Furthermore, the dispersion diagrams in Fig. 4 also indicate the emergence of a characteristic mode different from $\ell=1$ in the cases of bigger size parameters (see Fig. 4a), as well as of small values of the motility parameters $\sigma$ (see Fig. 4-c) and $\beta$ (see Fig. 4-d). Interestingly, the characteristic mode is not significantly affected by varying only the dimension of the external domain, while keeping the necrotic radius $R_{N}$ and the initial tumour radius $R_{T}$ fixed (Fig. 4-b) Moreover, whether the range of unstable modes is highly influenced by the sizes parameters and by the motility parameter $\sigma$ (Fig. 4 -a-c), it is not deeply influenced by variations of $R_{\text {out }}$ and $\beta$ (Fig. 4-b-d). Indeed as either the size of the domains decreases (Fig. 4-a) or $\sigma$ increases, the range of unstable modes decreases, up to a range where only $\ell=1$ is unstable. The dependency on the size of the domains states that smaller diffusive lengths (i.e. smaller diffusion coefficient or higher absorption rate of the nutrients) lead to highly irregular contours during the growth of the tumour. On the other hand, the effect on the mechanical parameter $\sigma$ on the dispersion diagram shows that, as expected, the surface tension $\sigma_{b}$, along with an high permeability of the surrounding porous environment $k$ act a stabilizing effect on the front (see Fig. 4-c), whereas the viscosity of the tumour cluster destabilize the border of the MCTS leading to more aggressive tumours. As $\beta$ settles the velocity of the quasi-stationary moving front (see Eq. (14)), the dispersion diagram in Fig. 4-d shows that the tumour developed highly irregular contour only in the case of slowly-moving front (i.e. small chemotactic coefficient and proliferation), since for fast moving front the characteristic mode decrease, until only $\ell=1$ is unstable.

Moreover, it is interesting to consider the role played by the radius of the growing tumour in the development of instabilities, while keeping all the other parameters fixed (see Fig. 5). Fig. 5-a reports the results for a set of parameters $R_{\text {out }}, R_{N}, \beta$ and $\sigma$ for which, independently from $R_{T}$, the most unstable mode is always $\ell=1$. This situation corresponds to a sort of translation of the spheroid inside the domain (see Fig. 5-a on the right). On the other hand, the characteristic mode depends on the MCTS size in a certain range of material parameters (see Fig. 5-b ). Indeed, it increases for increasing $R_{T}$, so that bigger tumours show more irregularities at their border. Therefore, sogrowing MCTS can undergo a morphological transition that may significantly affect the invasion pattern towards 


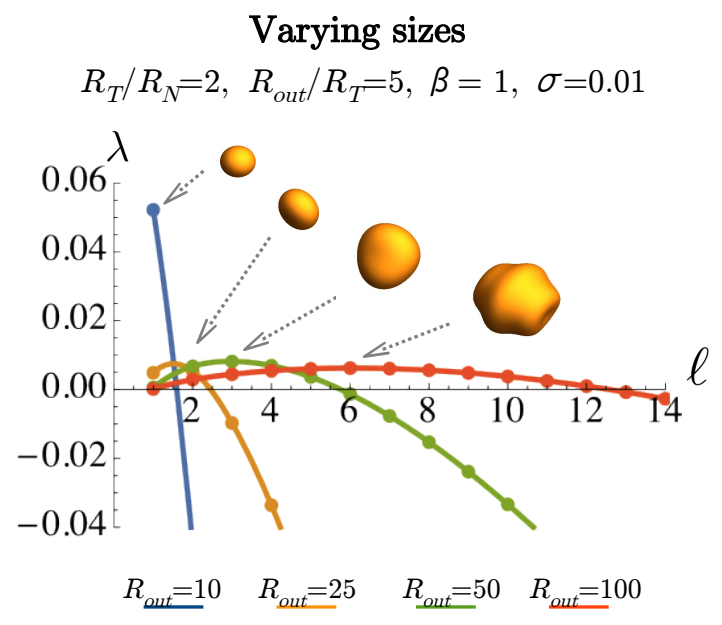

(a)

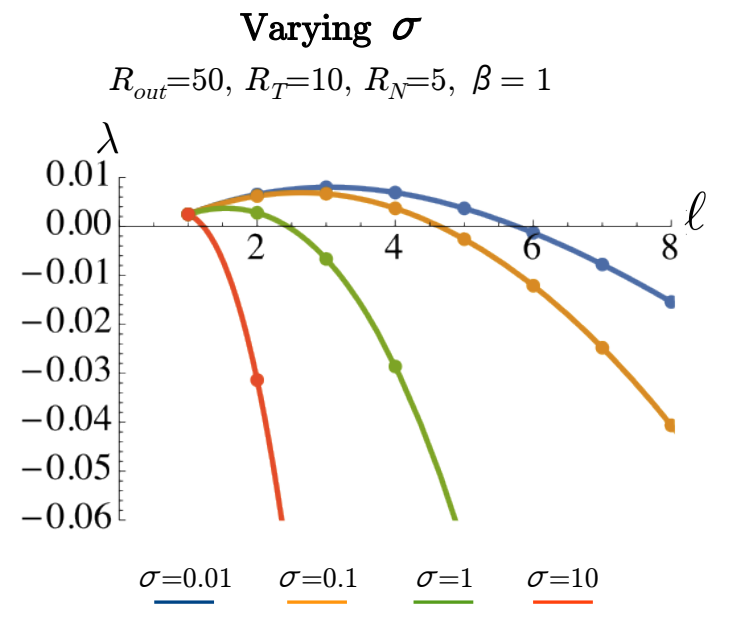

(c)

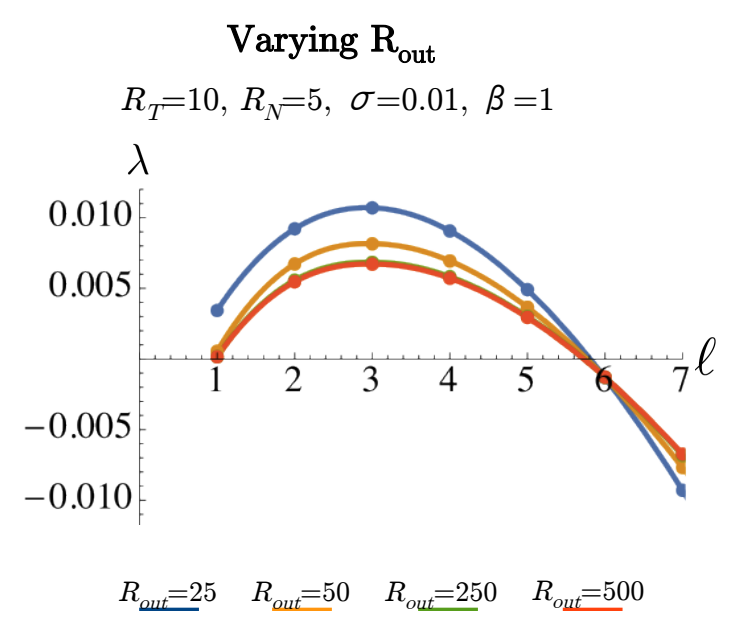

(b)

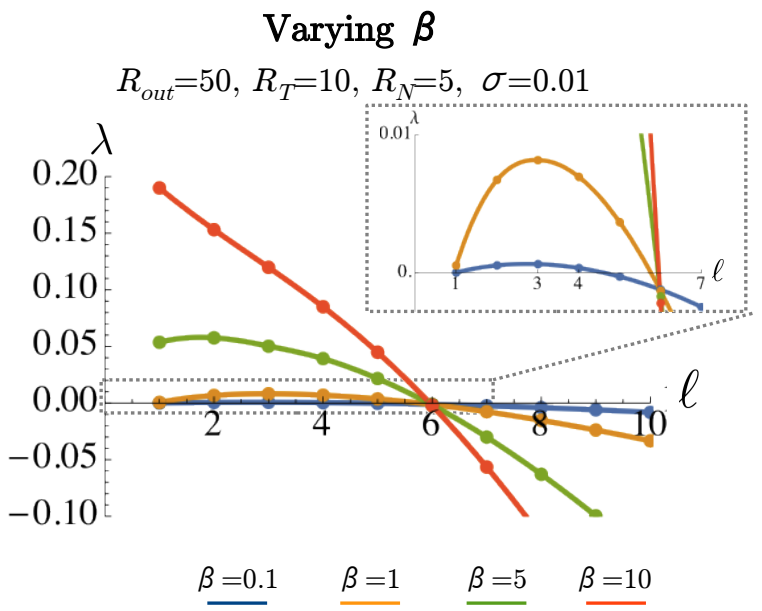

(d)

Figure 4. Dispersion diagrams for different values of the model parameters (a) $R_{\text {out }}$ keeping $q=R_{\text {out }} / R_{N}$ constant, (b) $R_{\text {out }}$ keeping $R_{N}$ constant, (c) $\sigma$ and (d) $\beta$. The solid lines in the graphs are obtained by interpolating the discrete values (see the dots on the curves) of the time growth rate of the perturbation, $\lambda$, calculated for integer values of $\ell$ from eq. (24). In (a) the shapes of the tumour corresponding to the characteristic mode $(\ell=1,2,3,6)$ is reported.

the typical finger-like structures observed for invasive carcinomas (see Fig. 5-b).

Finally, Fig. 6 depicts the perturbed pressure and velocity fields for a linearly unstable perturbation, given by a spherical harmonic of the kind $Y_{10}^{6}(\theta, \varphi)$. The highest variation of the pressure is located in a thin shell closer to the interface of the tumour, so that in the bulk of the tumour the velocity is almost null. In the region just at the rear of small protrusions (due to the perturbation of the boundary), the pressure field increases, so that the velocity at the border of the MCTS where a protrusion form, for the unstable modes (such as the one reported in Fig. 6), is higher than the velocity in the invagination on the contour. Furthermore, from the perturbed field ${ }^{447}$ is possible to appreciate small negative radial velocities in the bulk, just at the rear of the region where protrusion forms. Thus, while the spheroid surface moves outward, some cells inside the cluster move inward. This result confirms the existence of a radial convergent flow, in addition to the divergent flow that makes the aggregate expand, as pointed out in ${ }^{66,67}$. This effect combined with the higher velocity associated to the protrusion border could explain the possible detachment of carcinoma cells that lead to metastasis and thus the higher invasivity of tumours with irregular contours.

$\mathrm{E}^{30}$ ven though the onset of irregular contours and the development of a retrograde flow are in qualitative agree${ }_{\text {minent }}^{432}$ with biological experiments ${ }^{66-68}$, a direct quantita- 

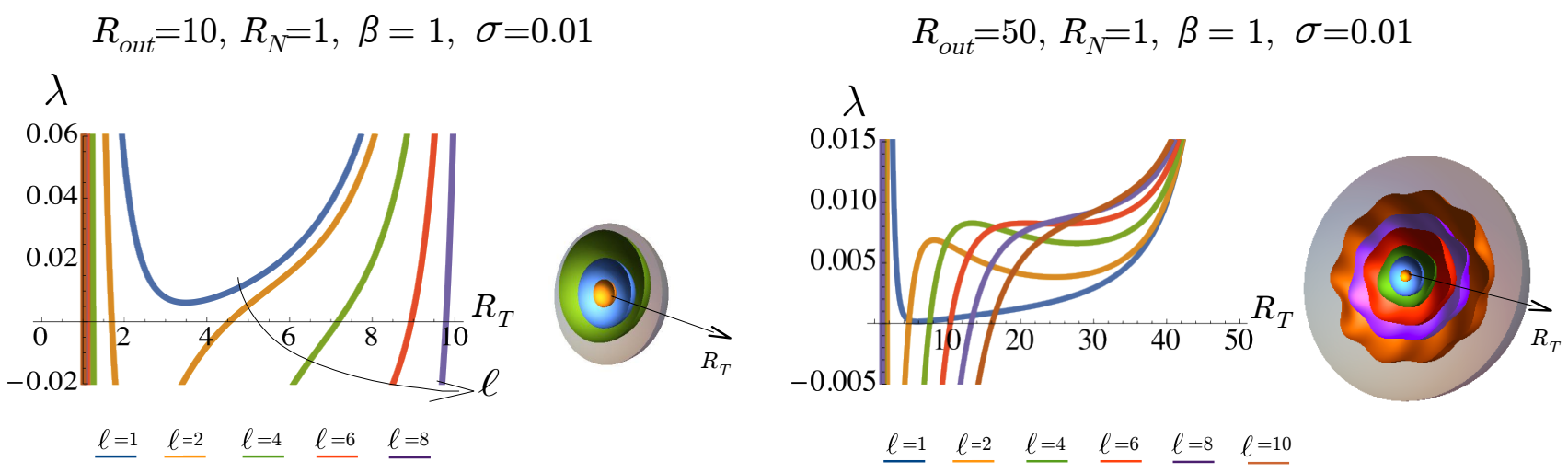

Figure 5. Evolution of the time growth rate of the perturbation $\lambda$ with respect to the dimensionless tumour radius, $R_{T}$, for different values of $\ell$ (with $\ell=1,2,4,6,8,10$ ). (a) For the chosen set of parameters, $\ell=1$ is the most unstable mode, whatever the tumour radius is. The deformed shapes corresponding to $R_{T}=2,5,8$ are reported aside (the gray region represents the outer environment). (b) The characteristic mode $\ell$ changes for different values of the tumour radius. Aside the dispersion curves, the section of the tumour perturbed shapes are reported for $R_{T}=2.5,8,12.5,20,26,35$ to which the corresponding characteristic modes are $\ell=1,2,4,6,8,10$ respectively.

tive comparison between our predictions and the biolog 83 ical experiments is not straightforward. First, not all the data required by the mathematical model, even thougdas measurable in principle, are reported in literature. Sec86 ond, most of the work in the vast literature on MCTSs focus on the effect of nutrients availability and stress on the growth of the spherical tumor aggregate, whereas lits9 tle attention have been paid on the systematic mapping ofo contour instabilities onset and evolution. Therefore, furer ther morphological data on MCTS, combined with estigr mates of the underlying biological parameters involved ias the process (i.e. nutrients diffusion and uptake, surface tension of the aggregate and permeability of the porouss medium), are highly required for the future validation offo the proposed model.

\section{Conclusions}

In this work we have presented a continuum model for describing the avascular growth of a multicellular tumour spheroid, comprising a fixed necrotic core surrounded by a region of proliferative cells, guided by the uptake of a diffusing nutrient. The proposed model encapsulates the diffusion of a chemical species from the vasculature of the healthy region and the tumour cell response to nutrients, via their proliferation and their chemotactic migration inside the extracellular space. The proposed model differs from existing approaches ${ }^{2,49,50}$ since it considers a growth though a rigid, porous surrounding material. Moreover, the MCTS expansion is guided not only by cell proliferation as in $^{2,49,50}$, but also by the chemotactic motion of cells, through a non-convective mass flux term. Differently from $^{2,50}$, that assumed a Gibbs-Thompson relation ${ }^{69}$ on the moving boundary for the chemical potential, we considered a mechanical effect in term of a surface tension at the MCTS outer boundary, leading to the Young-Laplace equation at the interface.
The proposed model is governed by five dimensionless parameters: two of them, $\beta$ and $\sigma$ are called motility parameters and representing the mechano-biology cues, the other three are denoted size parameters and are related to the typical sizes of the domains with respect to the diffusive length. The analytic results predicted the existence of a quasi-stationary radially-symmetric tumour configuration that is always linearly unstable to asymmetric perturbations involving spherical harmonics $Y_{\ell}^{m}(\theta, \phi)$, with the range of the unstable modes depending on the dimension of the domain with respect to the diffusive length and on the motility parameter $\beta$, related to the chemotactic growth of the tumour. We remark that, whilst a MCTS growing inside an infinite homogeneous domain is marginally stable, i.e. $\lambda=0$ for $\ell=1^{11,15,50}$, the proposed model is always linearly unstable, since translational symmetry is broken by considering a finite dimension of the surrounding media. Furthermore, differently from existing works ${ }^{2,8,9,14,15,17}$, the perturbation analysis is conducted here without neglecting the diffusion timescale in the unstable growth rate.

The analysis of the perturbed field also pointed out a possible mechanism that could lead to the detachment of metastasis from the primary tumour mass, based on the development of higher velocity at the border of the MCTS and a convergent flow inside where protrusions form. This mechanism could explain why the propensity for asymmetric invasion and the installation of irregular morphology characterize the growth of aggressive carcinomas in vivo. Thus, this approach has the potential to foster our understanding on the process of transition from the benign to the aggressive tumour stage and might provide also some indications for improving therapeutic treatments. Indeed, more blurred and irregular contours detected in vivo can be related to more malignant tumour, with respect to smoother and clearer contours that can be associated to benign carcinomas. 

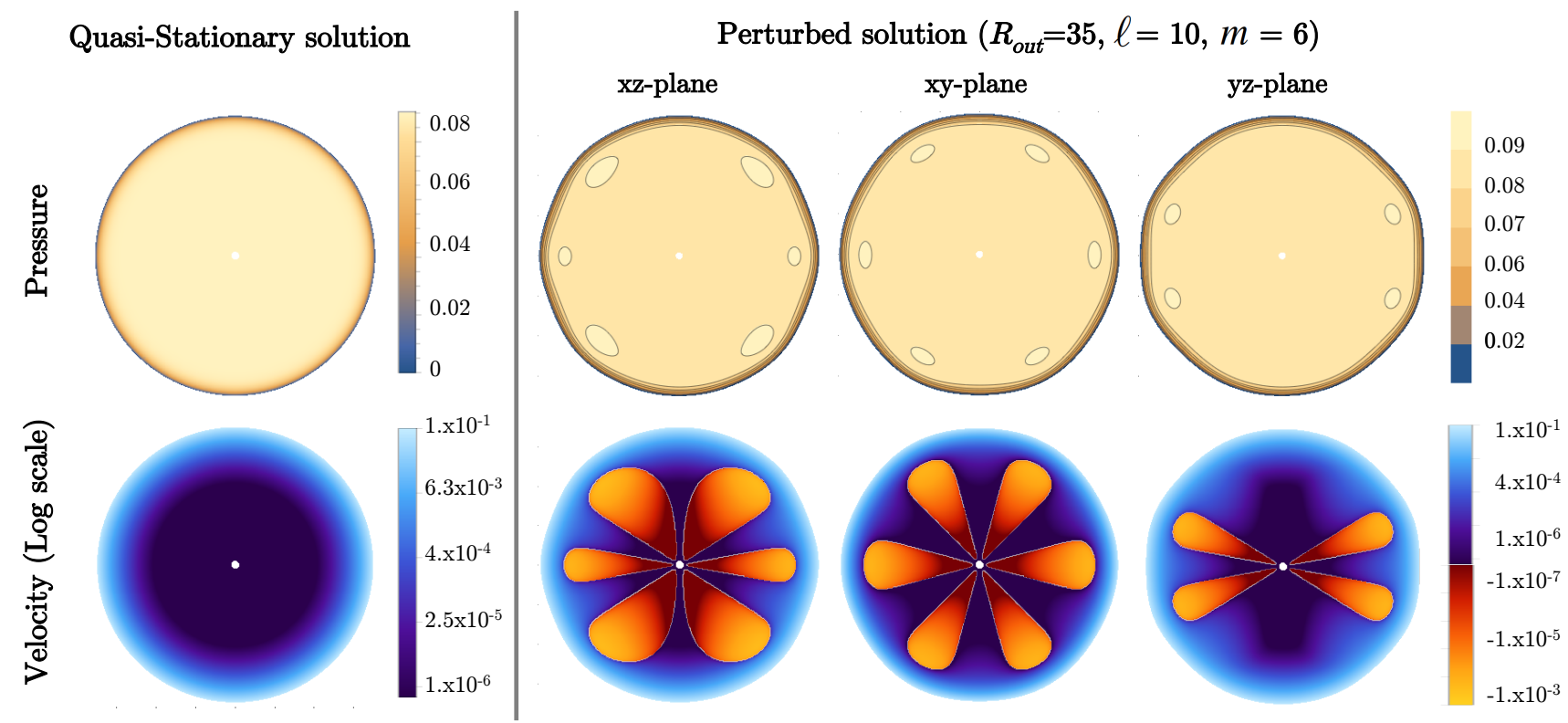

Figure 6. Evolution of the quasi-stationary and perturbed pressure and velocity fields for $R_{n}=1, R_{T}^{*}=35, R_{\text {out }}=50$ for a perturbation of the kind $e^{\lambda t} Y_{10}^{6}(\theta, \varphi)$. Since higher changes in the velocity and pressure field occurs only at the interface, we use a logarithmic scale in the velocity plot in order to show small variations of the perturbed field inside the bulk of the tumour. The perturbed velocity field highlights the existence of negative radial velocities (i.e. radial convergent flow), as pointed out in 66,67 .

However, the present model considers a really simplified geometry and adopts some simplifications in order to obtain a model that can be studied analytically. Thus, future improvements of the proposed mathematical model should focus on the explicit description of quiescent cell region (that in the present model corresponds to the regiost of the spheroid in which we have an almost null velocitysos and on tracking the evolution of the inner necrotic cor $\$$, occurring, for instance, when the nutrients concentratiosa attains a specific value ${ }^{2,12}$ (whereas in the present worki the fixed radius $R_{N}$ of the necrotic core is a parameter isa the sensitivity analysis). Then, the stability analysis casas be enriched by considering the weakly nonlinear interaø64 tions of the asymmetric modes, as well as their evolutiosas depending on the order $m$ of the spherical harmonic pe $\sigma_{6}$ turbation (as done for example in ${ }^{15}$ in a simplified casedr and numerical techniques should be developed in order t568 simulate the fully nonlinear evolution of the morphologita cal transition.

570 From the modelling point of view, future studies shoukd also consider the effect of the cells populating the sub ${ }^{2}$ rounding healthy environment on the consumption of nits trients and the effect of varying local densities (both insides the healthy tissue and inside the different tumor regions $\rangle_{5}$ on the nutrient diffusion coefficient. Then, the effect offo solid mechanical stresses on the growth dynamics of t $\mathrm{ta}_{77}$ mours ${ }^{32,61,67,70-73}$ and the effect of the possible deformars tion, degradation and reorganization of extracellular maz9 trix fibres ${ }^{74-76}$ should be included to move towards a moreo realistic representation of the problem. Indeed, for tumosi growing both in vivo and in xenograft animal models, thes description of the system evolution is far more complesx
Ełaan the one proposed in this work, referred to MCTS growth inside inert and rigid ECM scaffolds. In particuber, it has been shown that the geometrical and mechanical paroperties of the $\mathrm{ECM}^{74,75}$ play an important role for the zessible formation of metastasis, since they can lead to gुbowth arrest (i.e. spheroid compartmentalization) or, on Ełae contrary, foster the detachment of invasive cells. Along writh the rigidity of the matrix, its density, and the tenside forces generated in the $\mathrm{ECM}^{74,75}$, more recent studies bientify the matrix pore size as the critical property modskbating cancer cell invasion ${ }^{77,78}$. Based on these biological observations, some recent mathematical models have been developed to take into account, on one hand, MCTS søgregation by thick porous (but still rigid and homogeвъeous) structures ${ }^{79-81}$ and, on the other, ECM deformatiłon $^{82}$. Furthermore, not only ECM fibers can accumulate ${ }_{68} \mathrm{~b}$ being degraded at the host-MCTS interface, but they strongly reorganize, aligning parallel to the tumor borker, in a first stage, and then perpendicular to the tumor boundary ${ }^{74}$.

5thus, to take into account all these aspects and more ealistically describe tumor growth in vivo, an anisotropic @oro-elasto-visco-plastic model with a threshold (based on microscopic arguments) for cell motion should be developed. However in that case tumor irregular contours will bikely arise for inhomogeneity and anisotropy in the ECM, wwhereas this work demonstrates that mechano-biological and (macroscopic) geometrical cues can determine the ocgadrrence of a morphological transition in growing tumours Ełaat can promote invasiveness, even in an homogeneous eavironment. The theoretical results push towards the developments of further biological experiments for accu- 
rate characterization of MCTS morphology and careful measures of the surface tension and the interstitial pressure within MCTSs ${ }^{59,83}$, as well as growth and mobility properties of the tumour cells to validate the predictions of the model. Indeed, the integration of mathematical tools in biological research could be crucial for estimating the tumour's ability to invade its host environment.

\section{Acknowledgments}

This work was funded by the "Associazione Italiana per la Ricerca sul Cancro" (AIRC) through My First AIRC Grant 17412, partially supported by the "Start-up Packages and PhD Program" project, co-funded by Regione Lombardia through the "Fondo per lo sviluppo e la coesione 2007-2013 -formerly FAS", and by the "Progetto Giovani GNFM 2016".

\section{References}

1. J. Folkman and M. Hochberg, "Self-regulation of growth in three dimensions," The Journal of Experimental Medicine, vol. 138, pp. 745-753, 1973.

2. H. Byrne and M. Chaplain, "Free boundary value problems associated with the growth and development of multicellular spheroids," European Journal of Applied Mathematics, vol. 6, pp. 639-658, 1997.

3. R. Sutherland and R. Durand, "Growth and cellular characteristics of multicell spheroids," in Spheroids i68\% Cancer Research (H. Acker, J. Carlsson, R. Durand $d_{6}$ and R. M. Sutherland, eds.), vol. 95 of Recent Results;o in Cancer Research, pp. 24-49, Springer Berlin Heìi delberg, 1984.

4. K. Groebe and W. Mueller-Klieser, "Distributions of oxygen, nutrient and metabolic waste concentrations. in multicellular spheroids and their dependence oæs spheroid parameters," European Biophysics Journakto vol. 19, pp. 169-181, 1991.

5. R. Sutherland, "Cell and environment interactions i tumor microregions: the multicell spheroid model" Science, vol. 240 , no. 4849 , pp. 177-184, 1988.

6. J. Folkman, "Tumour angiogenesis," Advances i \& $_{1}$ Cancer Research, vol. 19, pp. 331-358, $1974 . \quad 682$

7. V. R. Muthukarruppan, L. Kubai, and R. Auerbach "Tumour-induced neovascularisation in the mouses eye," Journal of the National Cancer Institute, vol. $69_{85}$ pp. 699-705, 1982.

8. J. A. Adam, "A simplified mathematical model $ه f^{\prime}$ tumor growth," Mathematical Biosciences, vol. $81_{8}$ pp. 229-244, 1986.

9. J. A. Adam, "A mathematical model of tumour growth. ii. effects of geometry and spatial unifor $g_{g_{1}}$ mity on stability," Mathematical Biosciences, vol. 869, pp. $183-211,1987$.

10. J. A. Adam and S. A. Maggelakis, "Diffusion regldg4 lated growth characteristics of a spherical prevasculas carcinoma," Bulletin of Mathematical Biology, vol. 5296 pp. $549-582,1990$.
11. H. Byrne and M. A. J. Chaplain, "Growth of non-necrotic tumours in the presence and absence of inhibitors," Mathematical Biosciences, vol. 130, pp. 151-181, 1995.

12. H. Byrne and M. A. J. Chaplain, "Growth of necrotic tumours in the presence and absence of inhibitors," Mathematical Biosciences, vol. 135, pp. 187-216., 1996.

13. M. A. J. Chaplain, Experimental and Theoretical Advances in Biological Pattern Formation, ch. The development of a spatial pattern in a model for cancer growth., pp. 45-60. Plenum Press, 1993.

14. H. P. Greenspan, "Models for the growth of a solid tumour by diffusion," Studies in Applied Mathematics, vol. 52, pp. 317-340, 1972.

15. H. Byrne, "A weakly nonlinear analysis of a model of avascular solid tumour growth," Journal of Mathematical Biology, vol. 39, no. 1, pp. 59 - 89, 1999.

16. S. A. Maggelakis and J. A. Adam, "Mathematical model of prevascular growth of a spherical carcinoma," Mathematical and Computer Modelling, vol. 13, pp. 23-38, 1990.

17. D. L. S. McElwain and L. E. Morris, "Apoptosis as a volume loss mechanism in mathematical models of solid tumour growth," Mathematical Biosciences, vol. 39, pp. 147-157, 1978.

18. D. Ambrosi and L. Preziosi, "On the closure of mass balance models for tumor growth," Mathematical Models and Methods in Applied Sciences, vol. 12, no. 5, pp. 737-754, 2002.

19. R. P. Araujo and D. L. S. McElwain, "A history of the study of solid tumour growth: The contribution 610 of mathematical modeling," Bulletin of Mathematical ${ }_{611}$ Biology, vol. 66, pp. 1039-1091, 2004.

3อ. J. S. Lowengrub, H. B. Frieboes, F. Jin, Y. L. Chuang, ${ }_{613}$ X. Li, P. Macklin, S. M. Wise, and V. Cristini, "Non${ }_{614}$ linear modelling of cancer: Bridging the gap between 615 cells and tumours," Nonlinearity, vol. 23, pp. R1-R91, 6162010.

221. D. McElwain and G. Pettet, "Cell migration in mul${ }_{618}$ ticell spheroids: Swimming against the tide," Bulletin 619 of Mathematical Biology, vol. 55, no. 3, pp. 655-674, 6201993.

22. C. Chen, H. Byrne, and J. King, "The influence of 622 growth-induced stress from the surrounding medium ${ }_{623}$ on the development of multicell spheroids," Journal ${ }_{624}$ of Mathematical Biology, vol. 43, pp. 191-220, 2001.

23. K. A. Landman and C. P. Please, "Tumour dynamics 626 and necrosis: surface tension and stability," Mathe${ }_{627}$ matical Medicine and Biology, vol. 18, no. 2, pp. 131${ }_{628} 158,2001$.

224. M. Steinberg, "Reconstruction of tissues by dissoci630 ated cells. some morphogenetic tissue movements and ${ }_{631}$ the sorting out of embryonic cells may have a common ${ }_{632}$ explanation," Science, vol. 141, no. 3579, pp. 401-408, 6331963

235. R. Foty, G. Forgacs, C. Pflegerand, and M. Stein635 berg, "Liquid properties of embryonic tissues: Mea${ }_{636}$ surement of interfacial tensions," Physical Review Let- 
ters, vol. 72, no. 14, pp. 2298-2301, 1994.

26. G. Forgacs, R. Foty, Y. Shafrir, and M. Steinberg, "Viscoelastic properties of living embryonic tissues: a quantitative study," Biophysical Journal, vol. 74, no. 5, pp. 2227-2234, 1998.

27. G. Vitale and L. Preziosi, "A multhiphase model of tumor and tissue growth including cell adhesion and plastic reorganization," Mathematical Models and Methods in Applied Sciences, vol. 21, no. 9, pp. 19011932, 2011.

28. M. A. J. Chaplain and B. D. Sleeman, "Modelling the growth of solid tumours and incorporating a method for their classification using nonlinear elasticity theory," Journal of Mathematical Biology, vol. 31, pp. 431-473, 1993.

29. R. Skalak, S. Zargaryan, R. K. Jain, P. A. Netti, and A. Hoger, "Compatability and the genesis of residual stress by volumetric growth," Journal of Mathematical Biology, vol. 34, pp. 889-914, 1996.

30. D. Ambrosi and F. Mollica, "The role of stress in the growth of a multicell spheroid," Journal of Mathematical Biology, vol. 48, no. 5, pp. 477-499, 2004.

31. T. Roose, P. A. Netti, L. L. Munn, Y. Boucher, and R. K. Jain, "Solid stress generated by spheroid growth estimated using a linear poroelasticity model," $\mathrm{Mi}$ crovascular Research, vol. 66, no. 3, pp. 204-212, 2003.

32. C. Voutouri, F. Mpekris, P. Papageorgis, A. Odysseos, and T. Stylianopoulos, "Role of constitutive behavior and tumor-host mechanical interactions in the state of stress and growth of solid tumors," PLoS ONE, vol. 9, no. 8, p. e104717, 2014.

33. R. Vandiver and A. Goriely, "Morpho-elastodynamic\$s; The long-time dynamics of elastic growth," Journal off Biological Dynamics., vol. 3, pp. 180-195, 2009.

34. T. Stylianopoulos, J. D. Martin, V. P. Chauhan, S. Rвя Jain, B. Diop-Frimpong, N. Bardeesy, B. L. Smitheo C. R. Ferrone, F. J. Hornicek, Y. Boucher, L. L. Muniay and R. K. Jain, "Causes, consequences, and remedies for growth-induced solid stress in murine and humaঝs tumors," PNAS, vol. 109, no. 38, pp. 15101-15108 2012.

35. D. Ambrosi and L. Preziosi, "Cell adhesion mechaø6 nisms and stress relaxation in the mechanics of titor mours," Biomechanics and Modeling in Mechanobiodss ogy, vol. 8, pp. 397-413, 2009.

36. D. Ambrosi, L. Preziosi, and G. Vitale, "The interoo play between stress and growth in solid tumors," Mbor chanics Research Communications, vol. 42, pp. 87-9102 2012. Recent Advances in the Biomechanics of Growtabs and Remodeling.

37. C. Giverso, M. Scianna, and A. Grillo, "Growing avasos cular tumours as elasto-plastic bodies by thetheory ofo evolving natural configurations," Mechanics Researcabr Communications, vol. 68, pp. 31-39, 2015.

38. B. Aigouy, R. Farhadifar, D. Staple, A. Sagneag J. Röper, F. Jülicher, and S. Eaton, "Cell flow reorito ents the axis of planar polarity in the wing epithelium of drosophila," Cell, vol. 142, no. 5, pp. 773-786, 201912
39. T. Vasilica Stirbat, S. Tlili, T. Houver, J. P. Rieu, C. Barentin, and H. Delanoë-Ayari, "Multicellular aggregates: a model system for tissue rheology," The European Physical Journal E, vol. 36, no. 8, pp. 1-14, 2013.

40. J. Ranft, M. Basan, J. Elgeti, J. Joanny, J. Prost, and F. Jülicher, "Fluidization of tissues by cell division and apoptosis," PNAS, vol. 107, no. 49, pp. 20863$20868,2010$.

41. J. J. Casciari, S. V. Sotirchos, and R. M. Sutherland, "Mathematical modelling of microenvironment and growth in emt6/r0 multicellular tumour spheroids," Cell Proliferation, vol. 25, pp. 1-22, 1992.

42. M. Marusic, Z. Bajzer, J. P. Freyer, and S. VukPavlovic, "Analysis of growth of multicellular tumour spheroids by mathematical models," Cell Proliferation, vol. 27, pp. 73-94, 1994.

43. R. Muir, Muir's Textbook of Pathology. CRC Press, 15th edn ed., 2012.

44. S. S. Cross and D. W. K. Cotton, "The fractal dimension may be a useful morphometric discriminant in histopathology," The Journal of Pathology, vol. 166, pp. 409-411, 1992.

45. S. S. Cross, J. P. Bury, P. B. Silcocks, T. J. Stephenson, and D. W. K. Cotton, "Fractal geometric analysis of colorectal polyps," The Journal of Pathology, vol. 172 , pp. 317-323, 1994.

46. G. Landini and J. W. Rippin, "How important is tumour shape?," The Journal of Pathology, vol. 179, pp. 210-17, 1996.

427. R. Sutherland and R. E. Durand, "Radiation response 728 of multicell spheroids: An in vitro tumour model," 729 International Journal of Radiation Biology, vol. 23, 730 pp. 235-246, 1973.

48. M. Tubiana, "The kinetics of tumour cell proliferation 732 and radiotherapy," The British Journal of Radiology, 733 vol. 44 , pp. $325-347,1971$.

49. H. P. Greenspan, "On the growth and stability of cell 735 cultures and solid tumours," Journal of Theoretical 736 Biology, vol. 56, pp. 229-242, 1976.

50 . H. Byrne and M. Chaplain, "Modelling the role of 738 cell-cell adhesion in the growth and development of 739 carcinomas," Mathematical and Computer Modelling, 740 vol. 24 , no. 12 , pp. 1-17, 1996.

5ł1. M. Espinosa, G. Ceballos-Cancino, R. Callaghan, V. Maldonado, N. Patiño, V. Ruíz, and J. MeléndezZajgla, "Survivin isoform delta ex3 regulates tumor spheroid formation," Cancer Letters, vol. 318, no. 1, pp. $61-67,2012$.

52. A. Nyga, U. Cheema, and M. Loizidou, "3D tumour 74 models: novel in vitro approaches to cancer studies," 748 Journal of Cell Communication and Signaling, vol. 5, 749 pp. 239-248, 2011.

558. S. Wise, J. Lowengrub, H. Frieboes, and V. Cristini, 751 "Three-dimensional multispecies nonlinear tumor 752 growth-I Model and numerical method," Journal of 753 Theoretical Biology, vol. 253, no. 3, pp. 524-543, 2008. 554. A. Ramanathan, C. Wang, and S. Schreiber, "Perturbational profiling of a cell-line model of tumorigenesis 
63. P. Ciarletta, "Free boundary morphogenesis in liver ing matter," European Biophysics Journal, vol. 4103 pp. 681-686, 2012.

64. C. Giverso, M. Verani, and P. Ciarletta, "Branchingos instability in expanding bacterial colonies," Journ of The Royal Society Interface, vol. 12, no. 104, 201507

65. C. Giverso, M. Verani, and P. Ciarletta, "Emergø ing morphologies in round bacterial colonies: comparar ing volumetric versus chemotactic expansion," Biomer chanics and Modeling in Mechanobiology, pp. 1-19,1, 2015.

66. M. Dorie, R. Kallman, D. Rapacchietta1, D. Van Antwerp, and Y. Huang, "Migration anoth internalization of cells and polystyrene microspheвes in tumor cell spheroids," Experimental Cell Researchow vol. 141, no. 1, pp. 201-209, 1982.

67. M. Delarue, F. Montel, O. Caen, J. Elgeti, J. M $_{1}$ Siaugue, D. Vignjevic, J. Prost, J. F. Joanny, anolo G. Cappello, "Mechanical control of cell flow in multizo cellular spheroids," Physical Review Letters, vol. 1102, p. $138103,2013$.

68. H. Frieboes, X. Zheng, C.-H. Sun, B. Tromberg 23 R. Gatenby, and V. Cristini, "An integrated computazs tional/experimental model of tumor invasion," Canç25 Research, vol. 66, pp. 1597-1604, 2006.

69. J. Langer, "Instabilities and pattern formation in cry $\$ 27$ tal growth," Reviews of Modern Physics, vol. 52, no. $\mathbf{1}_{28}$ pp. 1-30, 1980 .

70. G. Cheng, J. Tse, R. Jain, and L. Munn, "Micrøзо environmental mechanical stress controls tumor spheroid size and morphology by suppressing proliferation and inducing apoptosis in cancer cells," PLoS $O N E$, vol. 4, no. 2, p. e4632, 2009.

71. F. Montel, M. Delarue, J. Elgeti, L. Malaquin, M. Basan, T. Risler, B. Cabane, D. Vignjević, J. Prost, G. Cappello, and J. F. Joanny, "Stress clamp experiments on multicellular tumor spheroids," Physical Review Letters, vol. 107, no. 18, p. 188102, 2011.

72. K. Alessandri, B. R. Sarangi, V. V. Gurchenkov, B. Sinha, T. R. Kie $\beta$ ling, L. Fetler, F. Rico, S. Scheuring, C. Lamaze, A. Simon, S. Geraldo, D. Vignjević, H. Doméjean, L. Rolland, A. Funfak, J. Bibette, N. Bremond, and P. Nassoy, "Cellular capsules as a tool for multicellular spheroid production and for investigating the mechanics of tumor progression in vitro," PNAS, vol. 110, no. 37, pp. 14843-14848, 2013.

73. R. K. Jain, J. D. Martin, and T. Stylianopoulos, "The role of mechanical forces in tumor growth and therapy," Annual review of biomedical engineering, vol. 16, pp. 321-346, 2014.

74. K. Kopanska, Y. Alcheikh, R. Staneva, D. Vignjevic, and T. Betz, "Tensile Forces Originating from Cancer Spheroids Facilitate Tumor Invasion," PLoS ONE, vol. 11, no. 6, p. e0156442, 2016.

75. P. Provenzano, K. Eliceiri, J. Campbell, D. Inman, J. White, and P. Keely, "Collagen reorganization at the tumor-stromal interface facilitates local invasion," $B M C$ Med, vol. 4, p. 38, 2006.

746. L. Kaufman, C. Brangwynne, K. Kasza, E. Filippidi, ${ }_{844}$ V. Gordon, T. Deisboeck, and D. Weitz, "Glioma Ex845 pansion in Collagen I Matrices: Analyzing Collagen 846 Concentration-Dependent Growth and Motility Pat847 terns," Biophysical Journal, vol. 89, p. 635650, 2005. 74. K. Wolf, M. Te Lindert, M. Krause, S. Alexander, ${ }_{849}$ J. Te Riet, A. Willis, R. Hoffman, C. Figdor, S. Weiss, 850 and P. Friedl, "Physical limits of cell migration: Con851 trol by ECM space and nuclear deformation and tun852 ing by proteolysis and traction force," The Journal of 853 Cell Biology, vol. 201, pp. 1069-1084, 2013.

758. A. Haeger, M. Krause, K. Wolf, and F. P., "Cell jam855 ming: collective invasion of mesenchymal tumor cells 856 imposed by tissue confinement," Biochimica et Bio${ }_{857}$ physica Acta, vol. 1840, no. 8, pp. 2386-95, 2014.

759. C. Giverso, A. Grillo, and L. Preziosi, "Influence of nu859 clear deformability on cell entry into cylindrical struc860 tures," Biomechanics and Modeling in Mechanobiol861 ogy, vol. 13, pp. 481-502, 2014.

860. A. Arduino and L. Preziosi, "A multiphase model of 863 tumour segregation in situ by a heterogeneous extra864 cellular matrix," International Journal of Non-Linear ${ }_{865}$ Mechanics, vol. 75, pp. 22-30, 2015.

82. C. Giverso, A. Arduino, and L. Preziosi, "How Nu867 cleus Mechanics and ECM Microstructure Influence 869 gates," [submitted], 2016.

\&2. G. Sciumé, R. Santagiuliana, M. Ferrari, P. Decuzzi, 871 and B. Schrefler, "A tumor growth model with de872 formable ECM," Physical Biology, vol. 11, no. 6, 
$932 \quad$ p. 065004, 2014.

933 83. R. K. Jain, "Barriers to drug delivery in solid tumours," Scientific American, vol. 271, no. 1, pp. 5865, 1994. 